\title{
Editorial Introduction to the Special Issue LOFT Sevilla
}

\author{
Giacomo Bonanno • Hans van Ditmarsch • \\ Wiebe van der Hoek
}

Received: 25 July 2013 / Accepted: 25 July 2013 / Published online: 25 August 2013

(C) Springer Science+Business Media Dordrecht 2013

This special issue of the Journal of Philosophical Logic contains a selection of papers presented at the 10th Conference on Logic and the Foundations of Game and Decision Theory (LOFT10), which took place at the University of Sevilla (Spain), July 18-20, 2012. While this special issue collects papers with a focus on logic, a second set of papers that are more game theory oriented can be found in a forthcoming special issue of the International Game Theory Review.

The LOFT conferences, which have taken place biannually since 1994, are interdisciplinary events that bring together researchers from a variety of fields: cognitive psychology, computer science and artificial intelligence, economics, game theory, linguistics, logic, philosophy and social choice. The central theme of the LOFT conferences has been the application of logic, in particular modal epistemic logic, to foundational issues in the theory of games and individual decision-making. For an overview of past and future LOFT events, please refer to http://www.econ.ucdavis. edu/faculty/bonanno/loft.html.

Game theory provides a formal language for the representation of interactive situations, that is, situations where several "entities" - called players - take actions

\footnotetext{
G. Bonanno $(\bowtie)$

Department of Economics, University of California, Davis, USA

e-mail: gfbonanno@ucdavis.edu

URL: http://www.econ.ucdavis.edu/faculty/bonanno/

H. van Ditmarsch

LORIA, CNRS - Université de Lorraine, Nancy, France

e-mail: hans.van-ditmarsch@loria.fr

URL: http://personal.us.es/hvd/

W. van der Hoek

Department of Computer Science, University of Liverpool, Liverpool, UK

e-mail:wiebe@csc.liv.ac.uk

URL: http://cgi.csc.liv.ac.uk/wiebe/
} 
that affect each other. The nature of the players varies depending on the context in which the game theoretic language is invoked: in evolutionary biology players are non-thinking living organisms; in computer science players are artificial agents; in behavioral game theory players are "ordinary" human beings, etc. Traditionally, however, game theory has focused on interaction among intelligent, sophisticated and rational individuals. A relatively recent development in game theory, the so-called epistemic foundation program, has sought to characterize, for any game, the behavior of rational and intelligent players who know the structure of the game and the preferences of their opponents and who recognize each other's rationality and reasoning abilities. The two fundamental questions addressed in this literature are: (1) Under what circumstances can a player be said to be rational? and (2) What does 'mutual recognition' of rationality mean? Since the two main ingredients of the notion of rationality are beliefs and choice and the natural interpretation of 'mutual recognition' of rationality is in terms of common belief, it is clear that the tools of epistemic logic are the appropriate tools for this program. The expression "interactive epistemology" has been used in the game-theory literature to refer to the analysis of strategic interaction based on an explicit modeling of the players' beliefs about each other's beliefs and rationality.

The LOFT conferences arose from the realization that the tools and methodology that were used in game theory were closely related to those used in other fields, notably computer science, logic and philosophy. Modal logic turned out to be the common language that made it possible to bring together different professional communities. It became apparent that the insights gained and the methodologies employed in one field could benefit researchers in other fields. Indeed, new and active areas of research have sprung from the interdisciplinary exposure provided by the LOFT events. Over time the scope of the LOFT conferences has broadened to encompass a wider range of topics, while maintaining its focus on the general issue of rationality and agency. Topics that have fallen within the LOFT umbrella include epistemic and temporal logic, theories of information processing and belief revision, counterfactual reasoning, models of bounded rationality, non-monotonic reasoning, theories of learning and evolution, social choice theory, etc.

This special issue contains papers that have a clear focus on logic and on the areas of reasoning where logics are applied. They reflect the general interests and the interdisciplinary scope of the LOFT research agenda. The four papers in this collection are contributions to defeasible reasoning, to (foundations of) practical reasoning, to belief and strategic reasoning, and to dynamic epistemic logic.

The paper 'Computing Strong and Weak Permissions in Defeasible Logic' by Guido Governatori, Francesco Olivieria, Antonino Rotolo and Simone Scannapieco proposes an extension of defeasible logic to represent and compute different concepts of defeasible permission. In particular, the authors discuss some types of explicit permissive norms that work as exceptions to opposite obligations or encode permissive rights. They show how strong permissions can be represented both with and without introducing a new consequence relation for inferring conclusions from explicit permissive norms. Finally, they illustrate how a preference operator applicable to contrary-to-duty obligations can be combined with a new operator representing ordered sequences of strong permissions. The logical system is 
studied from a computational standpoint and is shown to have linear computational complexity.

The paper 'Foundations for Everyday Practical Reasoning' by Hanti Lin is on practical reasoning. An example is 'Since today is Saturday, the grocery store is open today and will be closed tomorrow; so let's go today.' Everyday practical reasoning is reasoning directly with the propositions that one believes but that one may not be fully certain of. Everyday practical reasoning underpins one of our most familiar kinds of decisions. Foundational questions about it are largely ignored in standard decision theory. The authors addresses some such foundational questions. What are the decision rules in everyday practical reasoning that connect qualitative belief and desire to preference over acts? What sort of logic should govern qualitative beliefs in everyday practical reasoning, and to what extent is that logic necessary for the purposes of qualitative decisions? What kinds of qualitative decisions are always representable as results of everyday practical reasoning? Under what circumstances do the results of everyday practical reasoning agree with the Bayesian ideal of expected utility maximization? The paper proposes a rigorous decision theory for answering all of those questions, which is developed in parallel to Savage's foundation for expected utility maximization.

The paper 'Varieties of Belief in Strategic Interaction: a Logical Approach' by Emiliano Lorini proposes a logical framework for representing static and dynamic properties of different kinds of individual and collective attitudes. A complete axiomatization as well as a decidability result for the logic are given. The logic is applied to game theory by providing a formal analysis of the epistemic conditions of iterated deletion of weakly dominated strategies, or iterated weak dominance for short. The main difference between the analysis of the epistemic conditions of iterated weak dominance given in this paper and other analyses is that the author uses a semi-qualitative approach to uncertainty based on the notion of plausibility first introduced by Spohn, whereas other analyses are based on a quantitative representation of uncertainty in terms of probabilities.

The paper 'Action Emulation Between Canonical Models' by Floor Sietsma and Jan van Eijck investigates both Kripke models, used to model knowledge or belief in a static situation, and action models, used to model communicative actions that change this knowledge or belief. The appropriate notion for structural equivalence between modal structures such as Kripke models is bisimulation: Kripke models that are bisimilar are modally equivalent. The authors propose a structural relation called 'emulation' that plays the same role for the action models that play a prominent role in information updating. Two action models are equivalent if they yield the same results when updating Kripke models. More precisely, two action models are equivalent if it holds for all Kripke models that the result of updating with one action model is bisimilar to the result of updating with the other action model. Building on prior work involving some of the same authors, the current proposal contains a new notion of action emulation that characterizes the structural equivalence of the important class of canonical action models. Since every action model has an equivalent canonical action model, this gives a method to decide the equivalence of any pair of action models. The authors also give a partial result that holds for the class of all action models. 
The guest editors of this special issue would like to thank the authors for their submissions, the LOFT participants for their lively discussions and the many reviewers for their invaluable help during the thorough reviewing and editorial process. After LOFT, Hans van Ditmarsch has left the University of Sevilla for greener pastures. He would therefore like to use this occasion to thank the university and his colleagues there for their unfailing support during the conference and during his far too short stay in Sevilla. Last but not least, our thanks go to the Editors of the Journal of Philosophical Logic, for making this special issue possible. 